\title{
Quasi-PDFs and pseudo-PDFs
}

\section{Anatoly Radyushkin*}

Physics Department, Old Dominion University, Norfolk, Virginia 23529, USA

Thomas Jefferson National Accelerator Facility, Newport News, Virginia 23606, USA

E-mail: radyushejlab.org

We discuss the physical nature of quasi-PDFs, especially the reasons for the strong nonperturbative evolution pattern which they reveal in actual lattice gauge calculations. We argue that quasi-PDFs may be treated as hybrids of PDFs and the rest-frame momentum distributions of partons. The latter is also responsible for the transverse momentum dependence of TMDs. The resulting convolution structure of quasi-PDFs necessitates using large probing momenta $p_{3} \gtrsim 3$ $\mathrm{GeV}$ to get reasonably close to the PDF limit. To deconvolute the rest-frame distribution effects, we propose to use a method based directly on the coordinate representation. We treat matrix elements $M\left(z_{3}, p_{3}\right)$ as distributions $\mathscr{M}\left(v, z_{3}^{2}\right)$ depending on the Ioffe-time $v=p_{3} z_{3}$ and the distance parameter $z_{3}^{2}$. The rest-frame spatial distribution is given by $\mathscr{M}\left(0, z_{3}^{2}\right)$. Using the reduced Ioffe function $\mathfrak{M}\left(v, z_{3}^{2}\right) \equiv \mathscr{M}\left(v, z_{3}^{2}\right) / \mathscr{M}\left(0, z_{3}^{2}\right)$ we divide out the rest frame effects, including the notorious link renormalization factors. The $v$-dependence remains intact and determines the shape of PDFs in the small $z_{3}$ region. The residual $z_{3}^{2}$ dependence of the $\mathfrak{M}\left(v, z_{3}^{2}\right)$ is governed by perturbative evolution. The Fourier transform of $\mathscr{M}\left(v, z_{3}^{2}\right)$ produces pseudo-PDFs $\mathscr{P}\left(x, z_{3}^{2}\right)$ that generalize the light-front PDFs onto spacelike intervals. On the basis of these findings we propose a new method for extraction of PDFs from lattice calculations.

QCD Evolution 2017

22-26 May, 2017

Jefferson Lab Newport News, VA - USA

arXiv:1711.06031 [hep-ph]

JLAB-THY-17-2601

${ }^{*}$ Speaker. 


\section{Introduction}

The usual parton distribution functions (PDFs) $f(x)$ [1] measured in deep inelastic scattering and other inclusive processes are defined through matrix elements of certain bilocal operators on the light cone $z^{2}=0$. This fact prevents a direct extraction of these functions from Euclidean lattice gauge theory simulations. Still, recently, X. Ji [2] proposed to use separations $z=\left(0,0,0, z_{3}\right)$ which are purely space-like. Then one can define parton distributions in the $k_{3}=y p_{3}$ component of the parton momentum. These quasi-PDFs $Q\left(y, p_{3}\right)$ approach the light-cone PDFs $f(y)$ in the limit of large hadron momenta $p_{3} \rightarrow \infty$. The quasidistribution method can be also applied to distribution amplitudes (DAs). Lattice calculations of quasi-PDFs were discussed in Refs. [3, 4, 5]. The results for the pion quasi-distribution amplitudes (quasi-DAs) were reported in Ref. [6]. The lattice studies demonstrated a very strong change of quasidistributions with the probing momentum $p_{3}$, which cannot be explained by perturbative evolution.

In our recent papers [7,8], we have demonstrated that quasi-PDFs can be obtained from the transverse momentum dependent distributions (TMDs) $\mathscr{F}\left(x, k_{\perp}^{2}\right)$. We also showed that the $k_{\perp}^{2}$-dependence of TMDs plays the major role in the nonperturbative $p_{3}$-evolution of quasi-PDFs and quasi-DAs. As. In these papers, we have based our studies on the formalism of virtuality distribution functions $[9,10]$. The TMD/quasi-PDF relation allows to use simple models for TMDs for building models for the nonperturbative evolution of quasi-PDFs and quasi-DAs. The results obtained in our papers $[7,8]$ are in good agreement with the observed $p_{3}$-evolution patterns obtained in lattice calculations.

In the present talk, we outline the results and ideas formulated in our next paper [11]. First, it was demonstrated that the connection between TMDs and quasi-PDFs is, in fact, a mere consequence of Lorentz invariance. Thus, it may be derived in a much simpler way than in Ref. [7].

Then we show that the TMD/quasi-PDF connection formula may be rewritten in a form that allows a simple physical interpretation. Namely, it tells that when a hadron is moving, the parton $k_{3}$ momentum may be treated as coming from two sources. First, there is the motion of the hadron as a whole. It contributes the $x p_{3}$ part to the total $k_{3}$ value, and is governed by the dependence of the TMD $\mathscr{F}\left(x, \kappa^{2}\right)$ on its first, i.e., $x$, argument. The remaining part $\left(k_{3}-x p_{3}\right)$ comes from the rest-frame momentum distribution, and is governed by the dependence of the TMD on its second argument, $\kappa^{2}$. Thus, the quasi-PDFs may be treated as hybrids of PDFs and momentum distributions of partons in a hadron at rest.

Since $x$ appears in both arguments of the TMD, the quasi-PDFs have a convolution nature. This fact explains a rather complicated pattern of the change of quasi-PDFs with the probing momentum $p_{3}$, i.e., a strong nonperturbative $p_{3}$-evolution. One needs to have rather large values $p_{3} \sim 3 \mathrm{GeV}$ to "stop" the nonperturbative evolution and get sufficiently close to the PDF limit.

It should be emphasized that PDFs are given by the $k_{\perp}$ integral of the TMDs. Since our goal is to extract PDFs, information about a particular shape of the $k_{\perp}$-dependence is redundant. In a sense, one would prefer a situation when this $k_{\perp}$-dependence is given by a delta-function $\delta\left(k_{\perp}^{2}\right)$. Then the quasi-PDF $Q\left(y, p_{3}\right)$ would coincide with the PDF $f(y)$ for all probing momenta $p_{3}$. However, a physical TMD is a more involved function of $k_{\perp}$. What is worse, this irrelevant $k_{\perp}$-dependence of the TMDs results in a complicated structure of quasi-PDFs, necessitating large values of $p_{3}$ just to wipe out information about the $k_{\perp}$-dependence. One may ask if there are more economical ways 
of eliminating the unwanted $k_{\perp}$ effects.

The problem is that in TMD-based momentum representation, quasi-PDFs are given by a convolution of PDF-type $x$-dependence and $k_{\perp}$-dependence. The latter is related to the momentum distribution of the hadron at rest and basically reflects the finite size of the system. So, our next idea in Ref. [11] is that the deconvolution of the finite-size effects is much simpler in the coordinate representation. To this end, we introduce the functions $\mathscr{P}\left(x,-z^{2}\right)$ that we call pseudo-PDFs. They generalize the light-cone PDFs $f(x)$ onto spacelike intervals. In particular, one can take $z=\left(0,0,0, z_{3}\right)$. The $x$-dependence of the pseudo-PDFs is obtained through Fourier transforms of the Ioffe-time [12] distributions (ITDs) [13] $\mathscr{M}\left(v, z_{3}^{2}\right)$ with respect to $v=-(p z)$. It should be noted that the rest-frame momentum distribution is determined by $\mathscr{M}\left(0, z_{3}^{2}\right)$.

The ITDs are basically given by generic matrix elements like $M(z, p)=\langle p|\phi(0) \phi(z)| p\rangle$ which are the starting point of any lattice calculation. To have the ITD formulation, we should treat $M(z, p)$ as functions of $v=-(p z)$ and $z^{2}$ (or $v=p_{3} z_{3}$ and $z_{3}^{2}$ if we take $z=\left(0,0,0, z_{3}\right)$ ). The large- $z_{3}$ behavior of the pseudo-PDFs is governed by the same nonperturbative physics that determines the $k_{\perp}$-dependence of TMDs. To get PDFs, one should either take small $z_{3}$ directly, or extrapolate $\mathscr{P}\left(x, z_{3}^{2}\right)$ to small $z_{3}$ values. In this sense, taking small $z_{3}$ for pseudo-PDFs is analogous to taking large $p_{3}$ for quasi-PDFs.

However, a serious advantage of the pseudo-PDFs is that, unlike the quasi-PDFs, they have the "canonical" $-1 \leq x \leq 1$ support for all $z_{3}^{2}$. To access the $z_{3} \rightarrow 0$ limit through extrapolation, we propose to use the reduced pseudo-PDF $\mathfrak{P}\left(x, z_{3}^{2}\right) \equiv \mathscr{P}\left(x, z_{3}^{2}\right) / \mathscr{M}\left(0, z_{3}^{2}\right)$, in which the nonperturbative effects due to the rest-frame density are divided out. Thus, we argue that one should use the reduced ITD $\mathfrak{M}\left(v, z_{3}^{2}\right) \equiv \mathscr{M}\left(v, z_{3}^{2}\right) / \mathscr{M}\left(0, z_{3}^{2}\right)$ as the starting object for lattice calculations of PDFs. When $z_{3} \rightarrow 0$, the reduced ITDs obey the perturbative evolution equation, with $1 / z_{3}$ serving as an evolution scale parameter.

\section{Parton Distributions}

\subsection{Ioffe-time distributions and Pseudo-PDFs}

Studying hard processes, experimentalists work with hadrons. Theorists work with quarks. Thus, an important object is the amplitude $T(k, p)$ describing hadron-parton transition, with $p$ being the hadron momentum, and $k$ that of the quark. The transition can be described also using the coordinate space for quarks. Then we deal with the matrix element of a bilocal operator. We will write it in a generic form $\langle p|\phi(0) \phi(z)| p\rangle \equiv M(z, p)$ using scalar fields notations for quarks, since the basic concept of the parton distributions is not changed by spin complications.

By Lorentz invariance, the function $M(z, p)$ depends on $z$ through two scalar invariants, the Ioffe time [12] $(p z) \equiv-v$ and the interval $z^{2}$ (or $-z^{2}$ if we want a positive value for spacelike $z$ ):

$$
M(z, p)=\mathscr{M}\left(-(p z),-z^{2}\right) .
$$

The function $\mathscr{M}\left(v,-z^{2}\right)$ is the Ioffe-time distribution (ITD) [13].

It can be shown $[7,14]$ that, for all contributing Feynman diagrams, the Fourier transform of $\mathscr{M}\left(v,-z^{2}\right)$ with respect to $(p z)$ has the $-1 \leq x \leq 1$ support, i.e.,

$$
\mathscr{M}\left(v,-z^{2}\right)=\int_{-1}^{1} d x e^{-i x v} \mathscr{P}\left(x,-z^{2}\right) .
$$




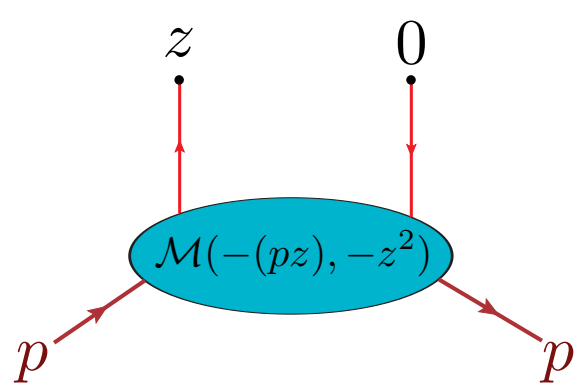

Figure 1: Ioffe-time distribution.

Note that Eq. (2.2) gives a covariant definition of $x$. There is no need to assume that $p^{2}=0$ or $z^{2}=0$ or take an infinite momentum frame, etc., to define $x$. As we will see, the function $\mathscr{P}\left(x,-z^{2}\right)$ generalizes the concept of the usual (or light-cone) parton distributions onto the case of non-lightlike intervals $z$. Following Ref. [11], we will call it pseudo-PDF. The $-1 \leq x \leq 1$ support region for pseudo-PDF is dictated by analytic properties of Feynman diagrams, and is determined by the structure of denominators of propagators. It is not affected by numerators present in nonscalar theories. The inverse transformation

$$
\mathscr{P}\left(x,-z^{2}\right)=\frac{1}{2 \pi} \int_{-\infty}^{\infty} d v e^{-i x v} \mathscr{M}\left(v,-z^{2}\right)
$$

may be treated as a direct definition of pseudo-PDFs as Fourier transforms of the ITDs $\mathscr{M}\left(v,-z^{2}\right)$ with respect to $v$ for fixed $z^{2}$. Thus, pseudo-PDFs stay just one step from the starting matrix element $M(z, p)$ (written in the form of ITD), and provide the most general object from which other parton distributions may be obtained as particular cases.

\subsection{Collinear Parton Distributions, Quasi-PDFs and TMDs}

Take a light-like $z$, say, that having just $z_{-}$component. Then $v=-p_{+} z_{-}$, and we can define the usual collinear (or light-cone) parton distribution $f(x)=\mathscr{P}(x, 0)$

$$
\mathscr{M}\left(-p_{+} z_{-}, 0\right)=\int_{-1}^{1} d x f(x) e^{-i x p_{+} z_{-}} .
$$

It has the usual interpretation of the probability that the parton carries fraction $x$ of the hadron's $p_{+}$momentum. Note that the $z^{2} \rightarrow 0$ limit is nontrivial in QCD and other renormalizable theories, since $\mathscr{M}\left(v, z^{2}\right)$ has $\sim \ln z^{2}$ singularities. The latter reflect perturbative evolution of parton densities. Within the operator product expansion approach (OPE), the $\ln z^{2}$ singularities are subtracted, e.g., by dimensional renormalization, and then $\ln \left(1 / z^{2}\right) \rightarrow \ln \mu^{2}$. Resulting PDFs depend on renormalization scale $\mu, f(x) \rightarrow f\left(x, \mu^{2}\right)$. If one keeps $z^{2}$ spacelike, then no subtractions are needed. For pseudo-PDFs $\mathscr{P}\left(x,-z^{2}\right)$, the interval $z^{2}$ serves as the ultraviolet (UV) cut-off, and $-1 / z^{2}$ is similar to the OPE scale $\mu^{2}$.

Taking a spacelike $z=\left\{0,0,0, z_{3}\right\}$ in the frame, where the hadron momentum is $p=\left(E, \mathbf{0}_{\perp}, P\right)$, one can define quasi-PDFs [2] as a Fourier transform of $M\left(z_{3}, P\right)$ with respect to $z_{3}$

$$
Q(y, P)=\frac{P}{2 \pi} \int_{-\infty}^{\infty} d z_{3} e^{-i y P z_{3}} M\left(z_{3}, P\right) .
$$


It is instructive to rewrite this integral in terms of the Ioffe-time distribution

$$
Q(y, P)=\frac{1}{2 \pi} \int_{-\infty}^{\infty} d v e^{-i y v} \mathscr{M}\left(v, v^{2} / P^{2}\right) .
$$

Unlike in the pseudo-PDF definition, the $v$-variable appears in both arguments of the ITD. We also see that $Q(y, P)$ tends to the usual PDF $f(y)$ in the $P \rightarrow \infty$ limit, as far as $\mathscr{M}\left(v, v^{2} / P^{2}\right) \rightarrow \mathscr{M}(v, 0)$.

The dependence of $\mathscr{M}\left(v,-z^{2}\right)$ on $v$ governs the $x$-dependence of $f(x)$, i.e. the longitudinal momentum structure of the hadron, while its $z^{2}$-dependence is directly connected with the transverse momentum distributions (TMDs). To show this, let us introduce TMDs. Take again the frame where $p=\left(E, \mathbf{0}_{\perp}, P\right)$, and choose $z$ that has $z_{+}=0$, nonzero $z_{-}$and, in addition, nonzero $z_{\perp}=\left\{z_{1}, z_{2}\right\}$ components. Then $z^{2}=-z_{\perp}^{2}$, and the TMD is defined by

$$
\mathscr{M}\left(v, z_{\perp}^{2}\right)=\int_{-1}^{1} d x e^{i x v} \int d^{2} k_{\perp} e^{-i\left(k_{\perp} z_{\perp}\right)} \mathscr{F}\left(x, k_{\perp}^{2}\right) .
$$

The parton again carries $x p_{+}$, but it also has transverse momentum $k_{\perp}$ which is Fourier-conjugate to $z_{\perp}$. Thus, the transverse momentum dependence of TMDs is governed by the $z^{2}$-dependence of ITDs. Note that, due to the rotational invariance in $z_{\perp}$ plane, this TMD depends on $k_{\perp}^{2}$ only.

While the quasi-PDF is derived from a matrix element involving purely "longitudinal" $z=z_{3}$, the dependence of $\mathscr{M}\left(v, z_{3}^{2}\right)$ on $z_{3}^{2}$ is given by the same function that defines the TMD by Eq. (2.7). To relate quasi-PDFs and TMDs, we take $z_{\perp}=\{0, v / P\}$ in Eq. (2.7) and substitute the resulting representation into the expression (2.6) for the quasi-PDF. This gives $[7,11]$

$$
Q(y, P)=P \int_{-1}^{1} d x \int_{-\infty}^{\infty} d k_{1} \mathscr{F}\left(x, k_{1}^{2}+(y-x)^{2} P^{2}\right) .
$$

According to this relation, the quasi-PDF variable $y$ has the $-\infty<y<\infty$ support, because the components of the transverse momentum $k_{\perp}$ in $\mathscr{F}\left(x, k_{\perp}^{2}\right)$ are not restricted.

\section{Structure of Quasi-PDFs}

\subsection{Momentum Distributions}

Since the variable $k_{1}$ is integrated over in Eq. (2.8), it makes sense to introduce the function

$$
\mathscr{R}\left(x, k_{3}\right) \equiv \int_{-\infty}^{\infty} d k_{1} \mathscr{F}\left(x, k_{1}^{2}+k_{3}^{2}\right)
$$

depending on the remaining momentum variable $k_{3}$ only (of course, according to Eq. (3.1), $\mathscr{R}\left(x, k_{3}\right)$ depends on $k_{3}$ through $k_{3}^{2}$. Also, instead of the quasi-PDFs $Q(y, P)$ that refer to the fraction $y \equiv$ $k_{3} / P$, one may consider distributions in the momentum $k_{3}$ itself: $R\left(k_{3}, P\right) \equiv Q\left(k_{3} / P, P\right) / P$. Then we can rewrite Eq. (2.8) as

$$
R\left(k_{3}, P\right)=\int_{-1}^{1} d x \mathscr{R}\left(x, k_{3}-x P\right) .
$$

For a hadron at rest, we have a one-dimensional function

$$
R\left(k_{3}, P=0\right) \equiv r\left(k_{3}\right)=\int_{-1}^{1} d x \mathscr{R}\left(x, k_{3}\right),
$$


that describes a primordial distribution of $k_{3}$ (or any other component of $\mathbf{k}$ ) in a rest-frame hadron. It may be directly obtained through a parameterization of the rest-frame density

$$
\mathscr{M}\left(0, z_{3}^{2}\right)=\int_{-\infty}^{\infty} d k_{3} r\left(k_{3}\right) e^{i k_{3} z_{3}} .
$$

According to Eq. (3.3), the rest-frame momentum distribution $r\left(k_{3}\right)$ is obtained from $\mathscr{R}\left(x, k_{3}\right)$ by taking the $x$-integral. Similarly, integrating $\mathscr{R}\left(x, k_{3}\right)$ over $k_{3}$ gives the collinear PDF

$$
\int_{-\infty}^{\infty} d k_{3} \mathscr{R}\left(x, k_{3}\right)=\int d^{2} k_{\perp} \mathscr{F}\left(x, k_{\perp}^{2}\right)=f(x) .
$$

Now we can give the following interpretation of the formula (3.2). According to it, in a moving hadron, the parton momentum $k_{3}=x P+\left(k_{3}-x P\right)$ has two parts. The $x P$ part comes from the motion of the hadron as a whole with the probability governed by $x$-dependence of $\mathscr{R}\left(x, k_{3}\right)$. The probability to get the remaining part $\left(k_{3}-x P\right)$ is governed by the dependence of $\mathscr{R}\left(x, k_{3}\right)$ on its second argument, $k_{3}$, associated with the primordial rest-frame momentum distribution.

\subsection{Factorized models for TMDs and quasi-PDFs}

Both arguments of $\mathscr{R}\left(x, k_{3}-x P\right)$ in Eq. (3.2) contain the integration parameter $x$. As a result, the shape of the momentum distributions $R(k, P)$ (and, hence, of the quasi-PDFs) is influenced by the form both of PDFs and rest-frame distributions. To illustrate the "hybrid" nature of momentum distributions and quasi-PDFs, we will use a factorized model $\mathscr{R}\left(x, k_{3}\right)=f(x) r\left(k_{3}\right)$. For the ITD, this Ansatz corresponds to the factorization assumption

$$
\mathscr{M}^{\mathrm{fact}}\left(v,-z^{2}\right)=\mathscr{M}(v, 0) \mathscr{M}\left(0,-z^{2}\right) .
$$

A popular choice is a Gaussian dependence of TMDs on $k_{\perp}$. It gives

$$
r_{G}\left(k_{3}\right)=\frac{1}{\sqrt{\pi} \Lambda} e^{-k_{3}^{2} / \Lambda^{2}} \text { or } r_{G}\left(z_{3}^{3}\right)=e^{-z_{3}^{2} \Lambda^{2} / 4} \text { for the rest frame density. }
$$

Then the factorized Gaussian model for the momentum distribution has the form

$$
R_{G}^{\mathrm{fact}}\left(k_{3}, P\right)=\frac{1}{\Lambda \sqrt{\pi}} \int_{-1}^{1} d x f(x) e^{-\left(k_{3}-x P\right)^{2} / \Lambda^{2}} .
$$

For PDF we choose a simple function $f(x)=4(1-x)^{3} \theta(0 \leq x \leq 1)$ resembling valence quark distributions. From Fig. 2, one can see that the curve for $R(k, P)$ changes from a Gaussian shape for small $P$ to a shape resembling a stretched PDF for large $P$. For small $P / \Lambda$ values, we may approximate

$$
R\left(k_{3}, P\right)=\int_{-1}^{1} d x f(x) r\left(k_{3}-x P\right) \approx r\left(k_{3}-\tilde{x} P\right)
$$

( $\tilde{x}=$ average $x$, in our model $\tilde{x}=0.2$ ), i.e., for small $P$, the $R\left(k_{3}, P\right)$ curve approximately keeps its shape, but the maximum shifts to the right when $P$ increases. For large $P$, we have

$$
r_{G}\left(k_{3}-x P\right)=\frac{1}{\sqrt{\pi} \Lambda} e^{-\left(k_{3}-x P\right)^{2} / \Lambda^{2}} \rightarrow \frac{1}{P} \delta\left(x-k_{3} / P\right),
$$

i.e., the combination $P R\left(k_{3}, P\right)$ corresponding to quasi-PDF $Q\left(y=k_{3} / P, P\right)$ in the large $P$ limit converts into a scaling function $f\left(k_{3} / P\right)=f(y)$ coinciding with the input PDF. 

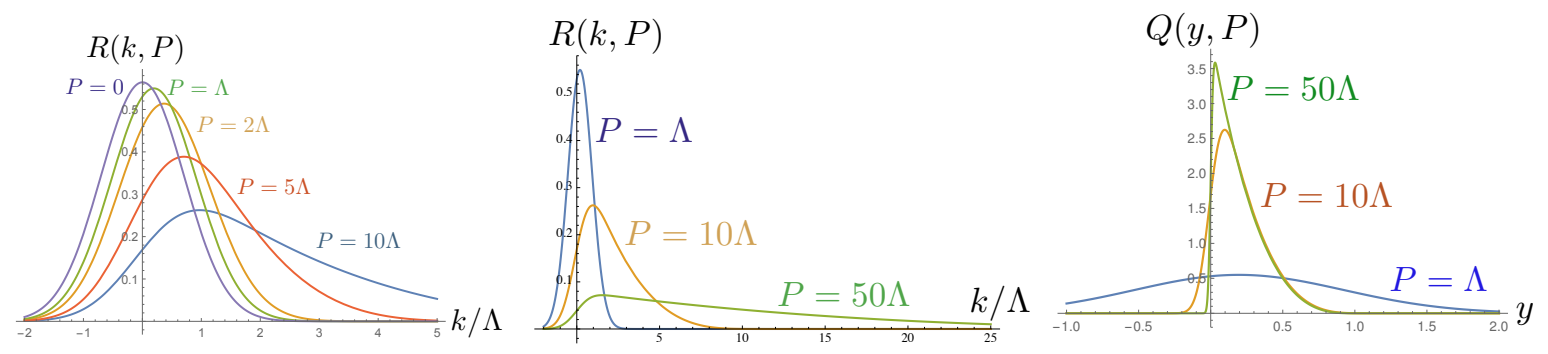

Figure 2: Momentum distribution $R(k, P)$ and quasi-PDF $Q(y, P)$ for different momentum $P$ values.

\subsection{QCD case}

In QCD we deal with matrix elements of the $\mathscr{M}^{\alpha}(z, p) \equiv\left\langle p\left|\bar{\psi}(0) \gamma^{\alpha} \hat{E}(0, z ; A) \psi(z)\right| p\right\rangle$ type, where $\hat{E}(0, z ; A)$ is the standard $0 \rightarrow z$ straight-line gauge link. Due to the vector index $\alpha$, the function $\mathscr{M}^{\alpha}(z, p)$ may be decomposed into $p^{\alpha}$ and $z^{\alpha}$ parts

$$
\mathscr{M}^{\alpha}(z, p)=2 p^{\alpha} \mathscr{M}_{p}\left(-(z p),-z^{2}\right)+z^{\alpha} \mathscr{M}_{z}\left(-(z p),-z^{2}\right) .
$$

In the standard definition of the TMD, we have $z_{+}=0$ and take $\alpha=+$. As a result, the $z^{\alpha}$-part drops out, and TMD $\mathscr{F}\left(x, k_{\perp}^{2}\right)$ is related to $\mathscr{M}_{p}\left(v, z_{\perp}^{2}\right)$ by the scalar formula. To remove the $z^{\alpha}$-contamination from quasi- PDF, we take the time component of $\mathscr{M}^{\alpha}\left(z=z_{3}, p\right)$ and define

$$
\mathscr{M}^{0}\left(z_{3}, p\right)=2 p^{0} \int_{-1}^{1} d y Q(y, P) e^{i y P z_{3}} .
$$

Then quasi-PDF $Q(y, P)$ is related to TMD $\mathscr{F}\left(x, k_{\perp}^{2}\right)$ by the scalar formula (2.8).

\section{Quasi-PDFs vs Pseudo-PDFs and Ioffe-time Distributions}

According to the definition of quasi-PDF $Q(y, P)$ in Eq.(2.6), they are obtained from the ITD $\mathscr{M}\left(v, z_{3}^{2}\right)$ by integration over $z_{3}=v / P$ lines in the $\left\{v, z_{3}\right\}$ plane, see Fig. 3. They tend to the horizontal $z_{3}=0$ line in the $P \rightarrow \infty$ limit, and the resulting quasi-PDFs approach PDF. It should

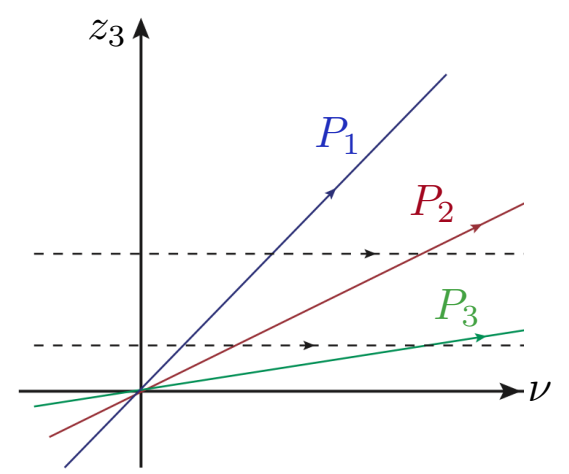

Figure 3: Lines of integration for quasi-PDF $Q(y, P)$ in the $\left\{v, z_{3}\right\}$ plane (solid lines) and for pseudo-PDFs $\mathscr{P}\left(x, z_{3}^{2}\right)$ (dashed lines). 
be noted that this approach is non-trivial, since $Q(y, P)$ has perturbative evolution with respect to $P$ for large $P$. In general, quasi-PDFs have the $-\infty<y<\infty$ support region. As we have seen, in the case of the soft factorized models, the support shrinks to $-1<y<1$ in the $P \rightarrow \infty$ limit. If one adds perturbative corrections due to hard gluon exchanges, they generate terms with the $-\infty<y<\infty$ support even in the $P \rightarrow \infty$ limit. Such terms should be removed through the use of matching conditions [2].

Pseudo-PDFs, according to their definition (2.3), are given by integration of $\mathscr{M}\left(v, z_{3}^{2}\right)$ over $z_{3}=$ const lines. They always have the $-1 \leq x \leq 1$ support. For small $z_{3}^{2}$, the pseudo-PDFs $\mathscr{P}\left(x, z_{3}^{2}\right)$ have perturbative evolution with respect to $1 / z_{3}$. At the leading logarithm level, they are close to usual PDFs $f\left(x, C^{2} / z_{3}^{2}\right)$ with $C$ being the matching coefficient, $C_{\overline{\mathrm{MS}}}=2 e^{-\gamma_{E}} \approx 1.12$.

The fact that quasi-PDFs $Q(y, P)$ are given by integration of $\mathscr{M}\left(v, z_{3}^{2}\right)$ over the $z_{3}=v / P$ lines leads to their $x$-convolution structure, even if $\mathscr{M}\left(v, z_{3}^{2}\right)$ factorizes, i.e., $\mathscr{M}\left(v, z_{3}^{2}\right)=\mathscr{M}(v, 0) \mathscr{M}\left(0, z_{3}^{2}\right)$. An alternative approach [11] is to convert lattice data for $\mathscr{M}\left(P z_{3}, z_{3}^{2}\right)$ into the data for $\mathscr{M}\left(v, z_{3}^{2}\right)$. The next step is to take the reduced function

$$
\mathfrak{M}\left(v, z_{3}^{2}\right) \equiv \frac{\mathscr{M}\left(v, z_{3}^{2}\right)}{\mathscr{M}\left(0, z_{3}^{2}\right)},
$$

i.e. divide ITD $\mathscr{M}\left(v, z_{3}^{2}\right)$ by the rest-frame density $\mathscr{M}\left(0, z_{3}^{2}\right)$. In factorized case, the reduced ITD converts into $\mathscr{M}(v, 0)$, and what formally remains is to take its Fourier transform to get PDF $f(x)$. Another advantage of using the reduced ITD is that the $z_{3}^{2}$-dependence due to self-energy of gauge link cancels in the ratio, because the UV-induced $z_{3}^{2}$-dependence is multiplicative (see Refs. $[15,16,17,18]$ for recent progress in this field.)

\subsection{Evolution of Ioffe-time distributions}

Originally, the Ioffe-time distributions $Q\left(v, \mu^{2}\right)$ were defined [13] as functions whose Fourier transforms with respect to $v$ were given by usual OPE PDFs $f\left(x, \mu^{2}\right)$. Thus, their dependence on the renormalization parameter $\mu$ (say, $\overline{\mathrm{MS}}$ scale) is completely determined by the evolution equation for PDFs $f\left(x, \mu^{2}\right)$. In case of pseudo-PDFs, the parameter $1 / z_{3}$ for small $z_{3}$ plays the role of $\mu$. A subtlety is that $\mathscr{M}\left(v, z_{3}^{2}\right)$ has an extra $z_{3}$ dependence induced by the renormalization of the gauge link. However, this $z_{3}$-dependence cancels in the reduced ITD $\mathfrak{M}\left(v, z_{3}^{2}\right)$. As a result, for small $z_{3}^{2}$ we have the leading-order evolution equation

$$
\frac{d}{d \ln z_{3}^{2}} \mathfrak{M}\left(v, z_{3}^{2}\right)=-\frac{\alpha_{s}}{2 \pi} C_{F} \int_{0}^{1} d u B(u) \mathfrak{M}\left(u v, z_{3}^{2}\right)
$$

with the same nonsinglet evolution kernel

$$
B(u)=\left[\frac{1+u^{2}}{1-u}\right]_{+}
$$

as in Ref. [13]. Examples of real and imaginary parts of ITD are shown in Fig. 4, together with functions $B \otimes \mathscr{M}$ governing their perturbative evolution. One can see that there are no perturbative evolution for $\mathscr{M}\left(0, z_{3}^{2}\right)$ [vector current is conserved]. Also, $\operatorname{Im} \mathscr{M}\left(0, z_{3}^{2}\right)=0$, i.e., the rest-frame distribution $\mathscr{M}\left(0, z_{3}^{2}\right)=0$ is purely real. 

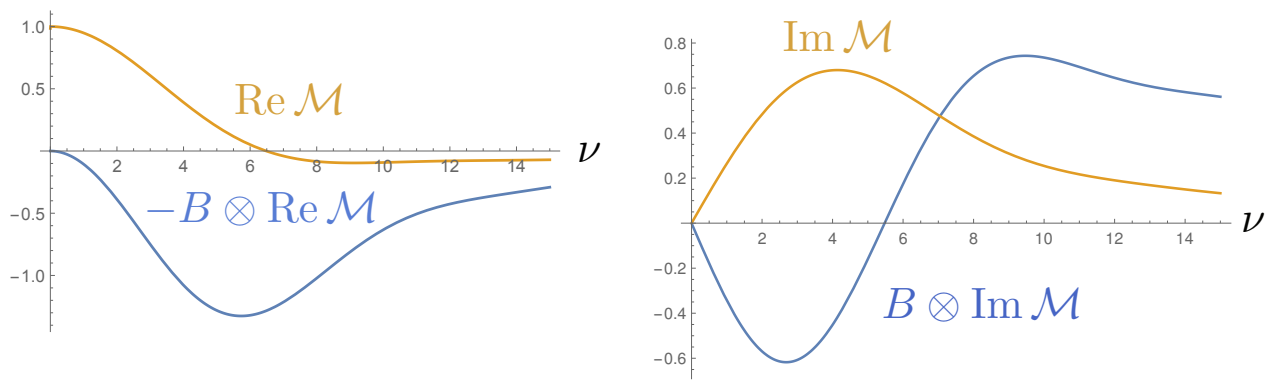

Figure 4: Real and imaginary parts of model Ioffe-time distribution $\mathscr{M}(v, 0)$ and the function $B \otimes \mathscr{M}$ governing their evolution.

\subsection{Nonfactorizable cases}

The perturbative evolution produces $z_{3}^{2}$-dependence for the reduced ITD $\mathfrak{M}\left(v, z_{3}^{2}\right)$. Hence, it will unavoidably violate factorization. This $z_{3}^{2}$-dependence should be visible in the data as $\ln \left(1 / z_{3}^{2} \Lambda^{2}\right)$ spikes for small $z_{3}^{2}$.

Take for illustration $\mathscr{P}^{\text {soft }}\left(x, z_{3}^{2}\right)=f(x) e^{-z_{3}^{2} \Lambda^{2} / 4}$ for the soft part (corresponding to the TMD $\left.\mathscr{F}^{\text {soft }}\left(x, k_{\perp}^{2}\right)=f(x) e^{-k_{\perp}^{2} / \Lambda^{2}} / \pi \Lambda^{2}\right)$ and choose $\alpha_{s} / \pi=0.1$ for the hard part in which we use the incomplete gamma-function $\Gamma\left[0, z_{3}^{2} \Lambda^{2} / 4\right]$ instead of a straightforward $\ln \left(1 / z_{3}^{2} \Lambda^{2}\right)$ function. In such a model for the hard part, the evolution stops for large $z_{3}^{2}$.

For the real part of the ITD, the evolution effects are the largest for $v \sim 6$. As one can see from Fig. 5, they are clearly visible for $z_{3} \Lambda \simeq 1.5$. Assuming $\Lambda=300 \mathrm{MeV}$, we obtain that $z_{3} \Lambda=1.5$ for $z_{3}=1 \mathrm{fm}$.

The reduced ITD $\mathfrak{M}\left(v, z_{3}^{2}\right)$ may also have residual $z_{3}^{2}$-dependence from the violation of factorization in the soft part. To illustrate these effects, we take the model pseudo-PDF $\mathscr{P}^{\text {soft }}\left(x, z_{3}^{2}\right)=$ $f(x) e^{-x(1-x) z_{3}^{2} \tilde{\Lambda}^{2} / 4}$ corresponding to the model TMD $\left.\mathscr{F}^{\text {soft }}\left(x, k_{\perp}^{2}\right)=f(x) e^{-k_{\perp}^{2} / x(1-x) \tilde{\Lambda}^{2}} /\left[\pi x(1-x) \tilde{\Lambda}^{2}\right]\right)$, whose dependence on $k_{\perp}^{2}$ comes through the $k_{\perp}^{2} / x(1-x)$ combination advocated by the light-front quantization proponents. To have the same $\left\langle k_{\perp}^{2}\right\rangle$ we need $\tilde{\Lambda}^{2}=\frac{15}{2} \Lambda^{2}$. The $z_{3}=1 \mathrm{fm}$ distance on the right graph of Fig. 5 corresponds to $z_{3}^{2} \Lambda^{2}=2.25$.
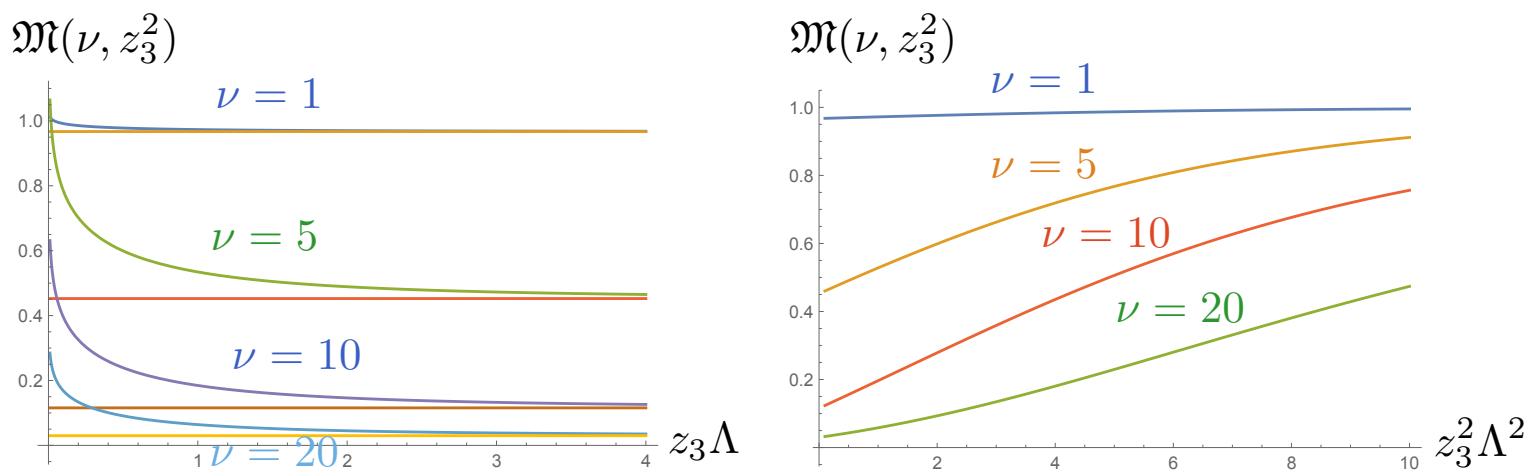

Figure 5: Violation of factorization for the reduced ITD due to perturbative evolution (left) and due to non-factorizable form of TMD (right). 
In a situation when factorization is violated both by evolution and non-perturbative effects, a possible strategy is to extrapolate $\mathfrak{M}\left(v, z_{3}^{2}\right)$ to $z_{3}^{2}=0$ from not too small values of $z_{3}^{2}$, say, from those above $0.5 \mathrm{fm}^{2}$. The resulting function $\mathscr{M}^{\text {soft }}(v, 0)$ may be treated as the Ioffe-time distribution producing the PDF $f_{0}(x)$ "at low normalization point". The remaining $\ln \left(1 / z_{3}^{2} \Lambda^{2}\right)$ spikes at small $z_{3}$ will generate its evolution.

\section{Summary}

In this talk, we described our recent work $[7,11]$ on the structure of parton quasi-distributions. We found that the quasi-PDFs are hybrids of PDFs and primordial rest-frame momentum distributions. The resulting complicated convolution nature of quasi-PDFs necessitates large probing momenta $p_{3} \gtrsim 3 \mathrm{GeV}$ to wipe out the primordial effects.

To avoid convolution structures, we proposed to use pseudo-PDFs $\mathscr{P}\left(x, z_{3}^{2}\right)$, the functions most closely related (by a Fourier transform) to the Ioffe-time distributions $\mathscr{M}\left(v, z_{3}^{2}\right)$, the primary objects both for continuum and lattice studies of parton distributions. One of the advantages of the pseudoPDFs is that they have the same "canonical" $-1 \leq x \leq 1$ support as usual PDFs. Furthermore, their $z_{3}^{2}$-dependence for small $z_{3}^{2}$ is governed by a usual evolution equation.

An important ingredient of the proposed program for the pseudo-PDF-based lattice extraction of PDFs is the use of the reduced Ioffe-time distributions given by the ratio $\mathscr{M}\left(v, z_{3}^{2}\right) / \mathscr{M}\left(0, z_{3}^{2}\right)$ in which the $z_{3}^{2}$-dependence of the primordial rest-frame density $\mathscr{M}\left(0, z_{3}^{2}\right)$ is divided out from the original Ioffe-time distribution $\mathscr{M}\left(v, z_{3}^{2}\right)$. The use of this ratio also provides a very simple and efficient way for getting rid of the $z_{3}^{2}$-dependence related to the ultraviolet divergences generated by the self-energy and vertex corrections to the gauge link.

While the write-up of this talk was in preparation, the exploratory lattice studies of the pseudoPDFs have been performed $[19,20]$. Their results have completely confirmed expectations formulated in Ref. [11].

\section{Acknowledgments}

I thank V. M. Braun, X. D. Ji and J.-W. Qiu for discussions and suggestions. I am especially grateful to K. Orginos for stimulating discussions and suggestions concerning the lattice implementation of the approach. This work is supported by Jefferson Science Associates, LLC under U.S. DOE Contract \#DE-AC05-06OR23177 and by U.S. DOE Grant \#DE-FG02-97ER41028.

\section{References}

[1] R. P. Feynman, Very high-energy collisions of hadrons, Phys. Rev. Lett. 23 (1969) 1415-1417.

[2] X. Ji, Parton Physics on a Euclidean Lattice, Phys. Rev. Lett. 110 (2013) 262002, [1305. 1539 ].

[3] H.-W. Lin, J.-W. Chen, S. D. Cohen and X. Ji, Flavor Structure of the Nucleon Sea from Lattice QCD, Phys. Rev. D91 (2015) 054510, [1402.1462].

[4] J.-W. Chen, S. D. Cohen, X. Ji, H.-W. Lin and J.-H. Zhang, Nucleon Helicity and Transversity Parton Distributions from Lattice QCD, Nucl. Phys. B911 (2016) 246-273, [1603. 06664 ]. 
[5] C. Alexandrou, K. Cichy, V. Drach, E. Garcia-Ramos, K. Hadjiyiannakou, K. Jansen et al., Lattice calculation of parton distributions, Phys. Rev. D92 (2015) 014502, [1 504 . 0 7455].

[6] J.-H. Zhang, J.-W. Chen, X. Ji, L. Jin and H.-W. Lin, Pion Distribution Amplitude from Lattice QCD, Phys. Rev. D95 (2017) 094514, [1702.00008].

[7] A. Radyushkin, Nonperturbative Evolution of Parton Quasi-Distributions, Phys. Lett. B767 (2017) 314-320, [1612.05170].

[8] A. V. Radyushkin, Pion Distribution Amplitude and Quasi-Distributions, Phys. Rev. D95 (2017) 056020, [1701.02688].

[9] A. V. Radyushkin, Virtuality Distributions in Application to $\gamma \gamma^{*} \rightarrow \pi^{0}$ Transition Form Factor at Handbag Level, Phys. Lett. B735 (2014) 417-425, [1404. 7032 ].

[10] A. V. Radyushkin, Virtuality and transverse momentum dependence of the pion distribution amplitude, Phys. Rev. D93 (2016) 056002, [1510.02517].

[11] A. V. Radyushkin, Quasi-parton distribution functions, momentum distributions, and pseudo-parton distribution functions, Phys. Rev. D96 (2017) 034025, [1705.01488].

[12] B. L. Ioffe, Space-time picture of photon and neutrino scattering and electroproduction cross-section asymptotics, Phys. Lett. 30B (1969) 123-125.

[13] V. Braun, P. Gornicki and L. Mankiewicz, Ioffe - time distributions instead of parton momentum distributions in description of deep inelastic scattering, Phys. Rev. D51 (1995) 6036-6051, [hep-ph/9410318].

[14] A. V. Radyushkin, On Spectral Properties of Parton Correlation Functions and Multiparton Wave Functions, Phys. Lett. 131B (1983) 179-182.

[15] T. Ishikawa, Y.-Q. Ma, J.-W. Qiu and S. Yoshida, Practical quasi parton distribution functions, 1609.02018.

[16] T. Ishikawa, Y.-Q. Ma, J.-W. Qiu and S. Yoshida, On the Renormalizability of Quasi Parton Distribution Functions, 1707.03107.

[17] X. Ji, J.-H. Zhang and Y. Zhao, Renormalization in Large Momentum Effective Theory of Parton Physics, 1706.08962 .

[18] J. Green, K. Jansen and F. Steffens, Nonperturbative renormalization of nonlocal quark bilinears for quasi-PDFs on the lattice using an auxiliary field, 1707.07152.

[19] K. Orginos, A. Radyushkin, J. Karpie and S. Zafeiropoulos, Lattice QCD exploration of parton pseudo-distribution functions, Phys. Rev. D96 (2017) 094503.

[20] J. Karpie, K. Orginos, A. Radyushkin and S. Zafeiropoulos, Parton distribution functions on the lattice and in the continuum, 1710.08288. 